\title{
Uniformly Geometric Functions Involving Constructed Operators
}

\section{Mohammad Al-Kaseasbeh and Maslina Darus}

School of Mathematical Sciences, Faculty of Science and Technology, Universiti Kebangsaan Malaysia, 43600 Bangi, Selangor, Malaysia

Correspondence should be addressed to Maslina Darus; maslina@ukm.edu.my

Received 22 February 2017; Accepted 28 March 2017; Published 16 April 2017

Academic Editor: Arcadii Z. Grinshpan

Copyright (c) 2017 Mohammad Al-Kaseasbeh and Maslina Darus. This is an open access article distributed under the Creative Commons Attribution License, which permits unrestricted use, distribution, and reproduction in any medium, provided the original work is properly cited.

This paper introduces classes of uniformly geometric functions involving constructed differential operators by means of convolution. Basic properties of those classes are studied, namely, coefficient bounds and inclusion relations.

\section{Introduction}

Throughout this paper, we are dealing with complex functions in the unit disc $\mathbb{U}=\{z \in \mathbb{C}:|z|<1\}$. More precisely, we are dealing with analytic functions of the form

$$
f(z)=z+\sum_{k=2}^{\infty} a_{k} z^{k}
$$

and we refer to them by $\mathscr{A}$.

The subordination between analytic functions $f(z)$ and $g(z)$ is written as $f(z) \prec g(z)$. Conceptually, the complex function $f(z)$ is subordinate to $g(z)$ if the image under $g(z)$ contains the images under $f(z)$. Technically, the complex function $f(z)$ is subordinate to $g(z)$ if there exists a Schwarz function $w$ with $w(0)=0$ and $|w(z)|<1$ for all $z \in \mathbb{U}$; such that

$$
f(z)=g(w(z)), \quad z \in \mathbb{U} .
$$

Let us consider the differential operators $R_{\alpha, \lambda}^{n}$ and $D_{\lambda}^{n}$ introduced, respectively, in $[1,2]$. Then, the convoluted operator of both of them is

$$
\begin{aligned}
\widetilde{D}_{\alpha, \lambda}^{n} f(z) & =D_{\lambda}^{n} f(z) * R_{\alpha, \lambda}^{n} f(z) \\
& =\left(z+\sum_{k=2}^{\infty}[1+\lambda(k-1)]^{n} a_{k} z^{k}\right)
\end{aligned}
$$

$$
\begin{aligned}
& *\left(z+\sum_{k=2}^{\infty}[1+\lambda(k-1)]^{n} C(\alpha, k) a_{k} z^{k}\right) \\
= & z+\sum_{k=2}^{\infty}[1+\lambda(k-1)]^{2 n} C(\alpha, k) a_{k}^{2} z^{k} .
\end{aligned}
$$

The operator $\widetilde{D}_{\alpha, \lambda}^{n}$ can also be written as

$$
\begin{aligned}
\widetilde{D}_{\alpha, \lambda}^{n} f(z)= & \underbrace{\varphi(z) * \cdots * \varphi(z)}_{2 n \text {-times }} * f(z) * \frac{z}{(1-z)^{\alpha+1}} \\
& * f(z) \\
= & \underbrace{\varphi(z) * \cdots * \varphi(z)}_{2 n \text {-times }} * f(z) * R^{\alpha} f(z),
\end{aligned}
$$

where

$$
\varphi(z)=\frac{z}{1-z}+\frac{\lambda z}{(1-z)^{2}}-\frac{\lambda z}{1-z} .
$$

A complex function $f \in \mathscr{A}$ is said to be in the class $\mathscr{C}(\eta)$ of convex functions of order $\eta$ in $\mathbb{U}$, if

$$
\mathfrak{R}\left\{1+\frac{z f^{\prime \prime}(z)}{f^{\prime}(z)}\right\}>\eta, \quad z \in \mathbb{U},
$$

where $\eta \in[0,1)$. 
On the other hand, a complex function $f \in \mathscr{A}$ is said to be in the class $\mathcal{S}^{*}(\eta)$ of starlike functions of order $\eta$ in $\mathbb{U}$, if

$$
\mathfrak{R}\left\{\frac{z f^{\prime}(z)}{f(z)}\right\}>\eta, \quad z \in \mathbb{U},
$$

where $\eta \in[0,1)$. The classes $\mathcal{S}^{*}(\eta)$ and $\mathscr{C}(\eta)$ are introduced in [3].

Notice that the classes $\mathcal{S}^{*} \equiv \mathcal{S}^{*}(0)$ and $\mathscr{C} \equiv \mathscr{C}(0)$ are the classical classes of starlike and convex functions in $\mathbb{U}$, respectively.

A complex function $f \in \mathscr{A}$ is said to be in the class of uniformly convex function of order $\eta$ and type $\zeta$, denoted by $\mathcal{U} \mathscr{C} \mathcal{V}(\zeta, \eta)$, if

$$
\Re\left\{1+\frac{z f^{\prime \prime}(z)}{f^{\prime}(z)}\right\}>\zeta\left|\frac{z f^{\prime \prime}(z)}{f^{\prime}(z)}\right|+\eta, \quad z \in \mathbb{U},
$$

where $\zeta \geq 0, \eta \in[0,1)$ and $\zeta+\eta \geq 0$, and is said to be in a corresponding class denoted by $\mathcal{S} \mathscr{P}(\zeta, \eta)$ if

$$
\mathfrak{R}\left\{\frac{z f^{\prime}(z)}{f(z)}\right\}>\zeta\left|\frac{z f^{\prime}(z)}{f(z)}-1\right|+\eta, \quad z \in \mathbb{U},
$$

where $\zeta \geq 0, \eta \in[0,1)$ and $\zeta+\eta \geq 0$. The classes $\mathcal{U} \mathscr{C} \mathscr{V}(\zeta, \eta)$ and $\mathcal{S} \mathscr{P}(\zeta, \eta)$ are introduced in [4].

The relation between classical starlike and convex functions, obviously, led us to the following relation.

$$
\begin{aligned}
f & \in \mathcal{U} \mathscr{C} \mathscr{V}(\zeta, \eta) \Longleftrightarrow \\
z f^{\prime} & \in \mathcal{S} \mathscr{P}(\zeta, \eta) .
\end{aligned}
$$

The classes $\mathcal{S} \mathscr{P}(\zeta, \eta)$ and $\mathscr{U} \mathscr{C} \mathscr{V}(\zeta, \eta)$ generalised other several classes. For $\zeta=0$, we obtain the classes $\mathcal{S}^{*}(\eta)$ and $\mathscr{C}(\eta)$, respectively. The class $\mathscr{U} \mathscr{C} \mathscr{V}(1,0) \equiv \mathscr{U} \mathscr{C} \mathscr{V}$ is known as the uniformly convex functions introduced in [5]. The class $\mathcal{S P}(1,0) \equiv \mathcal{S} \mathscr{P}$ is introduced in [6]. The classes $\mathscr{U} \mathscr{C} \mathscr{V}(1, \eta) \equiv \mathscr{U} \mathscr{C} \mathscr{V}(\eta)$ and $\mathcal{S} \mathscr{P}(1, \eta) \equiv \mathcal{S} \mathscr{P}(\eta)$ are investigated in [7]. For $\eta=0$, the classes $\mathscr{U} \mathscr{C} \mathscr{V}(\zeta, 0) \equiv$ $\zeta-\mathcal{U} \mathscr{C} \mathscr{V}$ and $\mathcal{S} \mathscr{P}(\zeta, 0) \equiv \zeta-\mathcal{S} \mathscr{P}$, respectively, are introduced in $[8,9]$.

Also, the classes $\mathcal{S} \mathscr{P}(\zeta, \eta)$ and $\mathscr{U} \mathscr{C} \mathscr{V}(\zeta, \eta)$ have been studied by Al-Oboudi and Al-Amoudi [10], involving certain differential operators.

\section{Geometric Interpretation}

The complex functions $f \in \mathcal{S} \mathscr{P}(\zeta, \eta)$ can be geometrically interpreted as follows.

$$
\begin{gathered}
f \in \mathcal{U} \mathscr{C} \mathscr{V}(\zeta, \eta) \Longleftrightarrow \\
1+\frac{z f^{\prime \prime}(z)}{f^{\prime}(z)} \text { lies in } R_{\zeta, \eta},
\end{gathered}
$$

where $R_{\zeta, \eta}$ is the conic domain included in the right half plane such that

$$
R_{\zeta, \eta}=\left\{u+i v: u>\zeta \sqrt{(u-1)^{2}+v^{2}}+\eta\right\} .
$$

On the other hand, the complex functions $f \in$ $\mathscr{U} \mathscr{C} \mathscr{V}(\zeta, \eta)$ can be geometrically interpreted as

$$
\begin{aligned}
& f \in \mathcal{S P}(\zeta, \eta) \Longleftrightarrow \\
& \frac{z f^{\prime}(z)}{f(z)} \text { lies in } R_{\zeta, \eta} .
\end{aligned}
$$

Denote by $\mathscr{P}\left(P_{\zeta, \eta}\right)(\zeta \geq 0,-1 \leq \eta<1)$ the class of functions $p$, such that $p \prec P_{\zeta, \eta}$ where $P$ denotes the class of positive real part functions in $\mathbb{U}$, and $p \in \mathscr{P}$. The function $P_{\zeta, \eta}$ provides a conformal mapping between the unit disc and the domain $R_{\zeta, \eta}$ such that $1 \in R_{\zeta, \eta}$ and where the boundary of $R_{\zeta, \eta}$ can be parameterised by

$$
\partial R_{\zeta, \eta}=\left\{u+i v: u^{2}=\left(\zeta \sqrt{(u-1)^{2}+v^{2}}+\eta\right)^{2}\right\} .
$$

By few steps of computations, $\partial R_{\zeta, \eta}$ appear as conic sections that are symmetrical around the real axis. Therefore, domain $R_{\zeta, \eta}$ is an ellipse for $\zeta>1$, a parabola for $\zeta=1$, a hyperbola for $0<\zeta<1$, and a right half plane $u>\eta$ for $\zeta=0$.

Involving the operator $\widetilde{D}_{\alpha, \lambda}^{n}$ given by (3), we introduce the following classes.

Definition 1. The complex functions $f \in \mathscr{A}$ and satisfying

$$
\mathfrak{R}\left\{1+\frac{z \widetilde{D}_{\alpha, \lambda}^{n} f^{\prime \prime}(z)}{\widetilde{D}_{\alpha, \lambda}^{n} f^{\prime}(z)}\right\}>\zeta\left|\frac{z \widetilde{D}_{\alpha, \lambda}^{n} f^{\prime \prime}(z)}{\widetilde{D}_{\alpha, \lambda}^{n} f^{\prime}(z)}\right|+\eta,
$$

$z \in \mathbb{U}$

is denoted by $\mathcal{U} \mathscr{C} \mathscr{V}_{\alpha, \lambda}^{n}(\zeta, \eta)$, where $\zeta \geq 0, \eta \in[0,1)$ and $\zeta+\eta \geq 0$.

On the other hand, we introduce the correspondence class of $\mathcal{S}_{\alpha, \lambda}^{n}(\zeta, \eta)$ as follows.

Definition 2. The complex functions $f \in \mathscr{A}$ and satisfying

$$
\Re\left\{\frac{z \widetilde{D}_{\alpha, \lambda}^{n} f^{\prime}(z)}{\widetilde{D}_{\alpha, \lambda}^{n} f(z)}\right\}>\zeta\left|\frac{z \widetilde{D}_{\alpha, \lambda}^{n} f^{\prime}(z)}{\widetilde{D}_{\alpha, \lambda}^{n} f(z)}-1\right|+\eta,
$$

$z \in \mathbb{U}$

is denoted by $\mathcal{S P}_{\alpha, \lambda}^{n}(\zeta, \eta)$, where $\zeta \geq 0, \eta \in[0,1)$ and $\zeta+\eta \geq$ 0 .

It is clear that the complex function $f \in \mathscr{U} \mathscr{C} \mathscr{V}_{\alpha, \lambda}^{n}(\zeta, \eta)$ if and only if $z f^{\prime} \in \mathcal{S} \mathscr{P}_{\alpha, \lambda}^{n}(\zeta, \eta)$ and that $\mathscr{U} \mathscr{C} \mathscr{V}_{\alpha, \lambda}^{n}(\zeta, \eta) \subseteq$ $\mathcal{S} \mathscr{P}_{\alpha, \lambda}^{n}(\zeta, \eta)$.

From (16) and (17), the complex functions $f \in$ $\mathcal{U} \mathscr{C} \mathscr{V}_{\alpha, \lambda}^{n}(\zeta, \eta)$ and $f \in \mathcal{S P}_{\alpha, \lambda}^{n}(\zeta, \eta)$ if and only if $1+z \widetilde{D}_{\alpha, \lambda}^{n} f^{\prime \prime}(z) / \widetilde{D}_{\alpha, \lambda}^{n} f^{\prime}(z)$ and $z \widetilde{D}_{\alpha, \lambda}^{n} f^{\prime}(z) / \widetilde{D}_{\alpha, \lambda}^{n} f(z)$, respectively, laying in the conic domain $R_{\zeta, \eta}$ given in (12). Indeed, the conic domain $R_{\zeta, \eta}$ is lying entirely in the right half plane, which allows us to write conditions (16) and (17) as follows.

$$
p \prec P_{\zeta, \eta}
$$



obtain

By virtue of (16) and (17) and the behavior of $R_{\zeta, \eta}$, we

$$
\begin{array}{r}
\Re\left\{1+\frac{z \widetilde{D}_{\alpha, \lambda}^{n} f^{\prime \prime}(z)}{\widetilde{D}_{\alpha, \lambda}^{n} f^{\prime}(z)}\right\}>\frac{\zeta+\eta}{1+\zeta}, \quad z \in \mathbb{U}, \\
\Re\left\{\frac{z \widetilde{D}_{\alpha, \lambda}^{n} f^{\prime}(z)}{\widetilde{D}_{\alpha, \lambda}^{n} f(z)}\right\}>\frac{\zeta+\eta}{1+\zeta}, \quad z \in \mathbb{U},
\end{array}
$$

which means that

$$
\begin{array}{r}
f \in \mathcal{U} \mathscr{C} \mathscr{V}_{\alpha, \lambda}^{n}(\zeta, \eta) \Longrightarrow \\
\widetilde{D}_{\alpha, \lambda}^{n} f \in \mathscr{C}\left(\frac{\zeta+\eta}{1+\zeta}\right) \subseteq \mathscr{C}, \\
f \in \mathcal{S}_{\alpha, \lambda}^{n}(\zeta, \eta) \Longrightarrow \\
\widetilde{D}_{\alpha, \lambda}^{n} f \in \mathcal{S}^{*}\left(\frac{\zeta+\eta}{1+\zeta}\right) \subseteq \mathcal{S}^{*} .
\end{array}
$$

Conditions (19) and (20) led to the following inclusion relations, respectively.

$$
\begin{aligned}
& \mathscr{U} \mathscr{C} \mathscr{V}_{\alpha, \lambda}^{n}(\zeta, \eta) \subseteq \mathscr{C}_{\alpha, \lambda}^{n}\left(\frac{\zeta+\eta}{1+\zeta}\right), \\
& \mathcal{S}_{\alpha, \lambda}^{n}(\zeta, \eta) \subseteq \mathcal{S}_{\alpha, \lambda}^{* n}\left(\frac{\zeta+\eta}{1+\zeta}\right) \text {. }
\end{aligned}
$$

\section{Uniformly Starlike Functions}

This section concerns the class $\mathcal{S}_{\alpha, \lambda}^{n}(\zeta, \eta)$ and its properties, namely, inclusion relation and coefficient bounds.

3.1. Inclusion Relation. In this subsection, we study the inclusion relations. The following lemmas pave the way for doing so.

Lemma 3 (see [11]). Let $f$ and $g$ be starlike of order $1 / 2$. Then so is $f * g$.

Lemma 4 (see [12]). Let $f$ and $g$ be univalent starlike of order $1 / 2$. Then, for every function $F \in \mathscr{A}$, we have

$$
\frac{f(z) * g(z) F(z)}{f(z) * g(z)} \in \overline{c o}(F(\mathbb{U})),
$$

where $\overline{c o}$ denotes the closed convex hull.

Lemma 5 (see [12]). Let $f$ and $g$, respectively, be in the classes $\mathscr{C}$ and $\mathcal{S}^{*}$. Then, for every function $F \in \mathscr{A}$, we have

$$
\frac{f(z) * g(z) F(z)}{f(z) * g(z)} \in \overline{c o}(F(\mathbb{U})) .
$$

Lemma 6 (see [13]). Let $a$ and $b$ be complex constants and $h$ univalent convex in $\mathbb{U}$ with $h(0)=c$ and

$$
\Re(a h(z)+b)>0 .
$$

Let $g(z)=c+\sum_{k=1}^{\infty} b_{k} z^{k}$ be analytic in $\mathbb{U}$. Then

$$
g(z)+\frac{z g^{\prime}(z)}{a g(z)+b} \prec h(z) .
$$

implies $g(z) \prec h(z)$.

Lemma 7. Let $R^{\alpha} f(z) \in \mathcal{S P}_{\alpha, \lambda}^{n}(\zeta, \eta)$ and

$$
\sum_{k=2}^{\infty} \frac{k^{2}}{C(\alpha, k)}\left|a_{k}\right|<1
$$

Then $f \in \mathcal{S}_{\alpha, \lambda}^{n}(\zeta, \eta)$.

Proof. Let $R^{\alpha} f(z) \in \mathcal{S}_{\alpha, \lambda}^{n}(\zeta, \eta)$. Then

$$
\frac{z\left(\widetilde{D}_{\alpha, \lambda}^{n} R^{\alpha} f\right)^{\prime}}{\widetilde{D}_{\alpha, \lambda}^{n} R^{\alpha} f}(\mathbb{U}) \subseteq R_{\zeta, \eta}
$$

and from (22) we see that $\widetilde{D}_{\alpha, \lambda}^{n} R^{\alpha} f(z) \in \mathcal{S}^{*}$. We can write $\widetilde{D}_{\alpha, \lambda}^{n} f(z)$ in terms of $\widetilde{D}_{\alpha, \lambda}^{n} R^{\alpha}$ as follows:

$$
\widetilde{D}_{\alpha, \lambda}^{n} f(z)=\left(R^{\alpha}\right)^{-1} f(z) * \widetilde{D}_{\alpha, \lambda}^{n} R^{\alpha} f(z),
$$

and, by convolution properties, we obtain

$$
z\left(\widetilde{D}_{\alpha, \lambda}^{n} f(z)\right)^{\prime}=\left(R^{\alpha}\right)^{-1} f(z) * z\left(\widetilde{D}_{\alpha, \lambda}^{n} R^{\alpha} f(z)\right)^{\prime} .
$$

Using Lemma 5 we obtain

$$
\begin{aligned}
& \frac{z\left(\widetilde{D}_{\alpha, \lambda}^{n} f(z)\right)^{\prime}}{\widetilde{D}_{\alpha, \lambda}^{n} f(z)}=\frac{\left(R^{\alpha}\right)^{-1} f(z) * z\left(\widetilde{D}_{\alpha, \lambda}^{n} R^{\alpha} f(z)\right)^{\prime}}{\left(R^{\alpha}\right)^{-1} f(z) * \widetilde{D}_{\alpha, \lambda}^{n} R^{\alpha} f(z)} \\
& =\frac{\left(R^{\alpha}\right)^{-1} f(z) *\left(z\left(\widetilde{D}_{\alpha, \lambda}^{n} R^{\alpha} f(z)\right)^{\prime} / \widetilde{D}_{\alpha, \lambda}^{n} R^{\alpha} f(z)\right) \widetilde{D}_{\alpha, \lambda}^{n} R^{\alpha} f(z)}{\left(R^{\alpha}\right)^{-1} f(z) * \widetilde{D}_{\alpha, \lambda}^{n} R^{\alpha} f(z)} \\
& \in \overline{c o}\left(\frac{z\left(\widetilde{D}_{\alpha, \lambda}^{n} R^{\alpha} f\right)^{\prime}}{\widetilde{D}_{\alpha, \lambda}^{n} R^{\alpha} f}(\mathbb{U})\right) \subseteq R_{\zeta, \eta} .
\end{aligned}
$$

Therefore, $f \in \mathcal{S}_{\alpha, \lambda}^{n}(\zeta, \eta)$.

Theorem 8. Let $0 \leq \lambda \leq(1+\zeta) /(1-\eta)$ and

$$
\sum_{k=2}^{\infty} \frac{k^{2}}{C(\alpha, k)}\left|a_{k}\right|<1
$$

Then

$$
\mathcal{S} \mathscr{P}_{\alpha, \lambda}^{n+1}(\zeta, \eta) \subseteq \mathcal{S} \mathscr{P}_{\alpha, \lambda}^{n}(\zeta, \eta) .
$$

Proof. Let $f(z) \in \mathcal{S}_{\alpha, \lambda}^{n+1}(\zeta, \eta)$. Then the geometric interpretation (18) can be written in the following subordination relation.

$$
\frac{z\left(\widetilde{D}_{\alpha, \lambda}^{n+1} f(z)\right)^{\prime}}{\widetilde{D}_{\alpha, \lambda}^{n+1} f(z)} \prec P_{\zeta, \eta} .
$$


By the definition of $\widetilde{D}_{\alpha, \lambda}^{n} f(z)$, we obtain

$$
\begin{aligned}
\widetilde{D}_{\alpha, \lambda}^{n+1} f(z)= & (1-\lambda) \widetilde{D}_{\alpha, \lambda}^{n} R^{\alpha} f(z) \\
& +\lambda z\left(\widetilde{D}_{\alpha, \lambda}^{n} R^{\alpha} f(z)\right)^{\prime} \\
= & \widetilde{D}_{\alpha, \lambda}^{n} R^{\alpha} f(z)-\lambda \widetilde{D}_{\alpha, \lambda}^{n} R^{\alpha} f(z) \\
& +\lambda z\left(\widetilde{D}_{\alpha, \lambda}^{n} R^{\alpha} f(z)\right)^{\prime}, \\
\left(\widetilde{D}_{\alpha, \lambda}^{n+1} f(z)\right)^{\prime}= & \left(\widetilde{D}_{\alpha, \lambda}^{n} R^{\alpha} f(z)\right)^{\prime}-\lambda\left(\widetilde{D}_{\alpha, \lambda}^{n} R^{\alpha} f(z)\right)^{\prime} \\
& +\lambda z\left(\widetilde{D}_{\alpha, \lambda}^{n} R^{\alpha} f(z)\right)^{\prime \prime} \\
& +\lambda\left(\widetilde{D}_{\alpha, \lambda}^{n} R^{\alpha} f(z)\right)^{\prime} \\
= & \left(\widetilde{D}_{\alpha, \lambda}^{n} R^{\alpha} f(z)\right)^{\prime} \\
& +\lambda z\left(\widetilde{D}_{\alpha, \lambda}^{n} R^{\alpha} f(z)\right)^{\prime \prime} .
\end{aligned}
$$

With the notation of $p(z)=z\left(\widetilde{D}_{\alpha, \lambda}^{n} R^{\alpha} f(z)\right)^{\prime} / \widetilde{D}_{\alpha, \lambda}^{n} R^{\alpha} f(z)$, we have

$$
\frac{z p^{\prime}(z)}{p(z)}=1-p(z)+\frac{z\left(\widetilde{D}_{\alpha, \lambda}^{n} R^{\alpha} f(z)\right)^{\prime \prime}}{\left(\widetilde{D}_{\alpha, \lambda}^{n} R^{\alpha} f(z)\right)^{\prime}} .
$$

Thus we obtain

$$
\frac{z\left(\widetilde{D}_{\alpha, \lambda}^{n+1} f(z)\right)^{\prime}}{\widetilde{D}_{\alpha, \lambda}^{n+1} f(z)}=p(z)+\frac{\lambda z p^{\prime}(z)}{(1-\lambda)+\lambda p(z)} .
$$

If $\lambda=0$, then from (35) and (38)

$$
R_{\alpha, \lambda}^{n} f(z) \in \mathcal{S}_{\alpha, \lambda}^{n}(\zeta, \eta)
$$

If $\lambda \neq 0$, we can write by (35) and (38)

$$
p(z)+\frac{1}{(1-\lambda) / \lambda+p(z)} \cdot z p^{\prime}(z) \prec P_{\zeta, \eta} .
$$

Thereby, Lemma 6 and condition (20) imply $p \prec P_{\zeta, \eta}$ for $0 \leq$ $\lambda \leq(1+\zeta) /(1-\eta)$, since $P_{\zeta, \eta}$ is univalent and convex in $\mathbb{U}$.

Thus, $R_{\alpha, \lambda}^{n} f(z) \in \mathcal{S}_{\alpha, \lambda}^{n}(\zeta, \eta)$. Therefore, $f(z) \in$ $\mathcal{S}_{\alpha, \lambda}^{n}(\zeta, \eta)$ by Lemma 7 .

Corollary 9. Let $0 \leq \lambda \leq(1+\zeta) /(1-\eta)$ and

$$
\sum_{k=2}^{\infty} \frac{k^{2}}{C(\alpha, k)}\left|a_{k}\right|<1
$$

Then

$$
\mathcal{S} \mathscr{P}_{\alpha, \lambda}^{n}(\zeta, \eta) \subseteq \mathcal{S} \mathscr{P}_{\alpha, \lambda}(\zeta, \eta)
$$

Proof. The result is obtained by using Theorem 8 .
Remark 10. Considering the parameters $n, \alpha$, and $\zeta$ by certain values, new results are obtained as follows.

(1) Consider $\alpha=0$ in Theorem 8; we obtain, for $0 \leq \lambda \leq$ $(1+\zeta) /(1-\eta)$

$$
\mathcal{S P}_{\lambda}^{n+1}(\zeta, \eta) \subseteq \mathcal{S} \mathscr{P}_{\lambda}^{n}(\zeta, \eta)
$$

(2) Consider $\zeta=0$ in Theorem 8; we obtain, for $0 \leq \lambda \leq$ $(1+\zeta) /(1-\eta)$

$$
\mathcal{S}_{\alpha, \lambda}^{* n+1}(0, \eta) \subseteq \mathcal{S}_{\alpha, \lambda}^{* n}(0, \eta)
$$

Paving the way to prove next theorem, we provide the forthcoming lemma.

Lemma 11. If the complex function $f \in \mathcal{S}_{\alpha, \lambda}^{n}(\zeta, \eta)$, then $\widetilde{D}_{\alpha, \lambda}^{n} f(z) \in \mathcal{S}^{*}$ whenever $\zeta$ and $\eta$ lie, respectively, in $[0,1)$ and $[1 / 2,1)$ or $[0, \infty)$ and $[0,1)$.

Proof. The results follows immediately from (20) where $(\zeta+$ $\eta) /(1+\zeta) \geq 1 / 2$ under the restriction of the value of $\zeta$ and $\eta$.

Theorem 12. Let $0 \leq \mu \leq \alpha<1$ and

$$
\sum_{k=2}^{\infty} \frac{k^{2} C(\mu, k)}{C(\alpha, k)}\left|a_{k}\right|<1
$$

Then

$$
\mathcal{S}_{\alpha, \lambda}^{n}(\zeta, \eta) \subseteq \mathcal{S} \mathscr{P}_{\mu, \lambda}^{n}(\zeta, \eta)
$$

where $[(0 \leq \zeta<1$ and $1 / 2 \leq \eta)$ or $(\zeta \geq 1$ and $0 \leq \eta<1)]$.

Proof. Let $f \in \mathcal{S}_{\alpha, \lambda}^{n}(\zeta, \eta)$. Then by the definition of $\widetilde{D}_{\alpha, \lambda}^{n}$ and the convolution properties, we have

$$
\begin{aligned}
\widetilde{D}_{\mu, \lambda}^{n} f(z)= & \frac{z}{(1-z)^{\mu+1}} *\left(R^{\alpha}\right)^{-1} f(z) * f(z) \\
& * \underbrace{\varphi * \cdots * \varphi}_{2 n \text {-times }} * \frac{z}{(1-z)^{\alpha+1}} * f(z) \\
= & \frac{z}{(1-z)^{\mu+1}} *\left(R^{\alpha}\right)^{-1} * f(z) \\
& * \widetilde{D}_{\alpha, \lambda}^{n} f(z),
\end{aligned}
$$

$$
\begin{aligned}
z\left(\widetilde{D}_{\mu, \lambda}^{n} f(z)\right)^{\prime}= & \frac{z}{(1-z)^{\mu+1}} *\left(R^{\alpha}\right)^{-1} * f(z) \\
& * z\left(\widetilde{D}_{\alpha, \lambda}^{n} f(z)\right)^{\prime} .
\end{aligned}
$$


By Lemma 11 we have $\widetilde{D}_{\alpha, \lambda}^{n} f(z) \in \mathcal{S}^{*}(1 / 2)$. Using Lemma 4, we obtain

$$
\begin{aligned}
\frac{z\left(\widetilde{D}_{\mu, \lambda}^{n} f(z)\right)^{\prime}}{\widetilde{D}_{\mu, \lambda}^{n} f(z)} & =\frac{z /(1-z)^{\mu+1} *\left(R^{\alpha}\right)^{-1} f(z) * f(z) * z\left(\widetilde{D}_{\alpha, \lambda}^{n} f(z)\right)^{\prime}}{z /(1-z)^{\mu+1} *\left(R^{\alpha}\right)^{-1} f(z) * f(z) * \widetilde{D}_{\alpha, \lambda}^{n} f(z)} \\
& =\frac{z /(1-z)^{\mu+1} *\left(R^{\alpha}\right)^{-1} f(z) * f(z) *\left(z\left(\widetilde{D}_{\alpha, \lambda}^{n} f(z)\right)^{\prime} / \widetilde{D}_{\alpha, \lambda}^{n} f(z)\right) \widetilde{D}_{\alpha, \lambda}^{n} f(z)}{z /(1-z)^{\mu+1} *\left(R^{\alpha}\right)^{-1} f(z) * f(z) * \widetilde{D}_{\alpha, \lambda}^{n} f(z)} \\
& \in \overline{c o}\left(\frac{z\left(\widetilde{D}_{\alpha, \lambda}^{n} f\right)^{\prime}}{\widetilde{D}_{\alpha, \lambda}^{n} f}(\mathbb{U})\right) \subseteq R_{\zeta, \eta} .
\end{aligned}
$$

Therefore, $f \in \mathcal{S}_{\mu, \lambda}^{n}(\zeta, \eta)$.

Corollary 13. Let $\mu=0$. Also let $[(0 \leq \zeta<1$ and $1 / 2 \leq \eta)$ or $(\zeta \geq 1$ and $0 \leq \eta<1)]$ and

$$
\sum_{k=2}^{\infty} \frac{k^{2}}{C(\alpha, k)}\left|a_{k}\right|<1 .
$$

Then

$$
\mathcal{S}_{\alpha, \lambda}^{n}(\zeta, \eta) \subseteq \mathcal{S} \mathscr{P}_{\lambda}^{n}(\zeta, \eta)
$$

Proof. The results follows by Theorem 12 .

Remark 14. Considering the parameters $n, \alpha, \lambda$, and $\zeta$ by certain values, new results are obtained as follows.

(1) Consider $n=1$ and $\lambda=0$ in Theorem 12; we obtain, for $0 \leq \mu \leq \alpha<1$,

$$
\mathcal{S} \mathscr{P}_{\alpha, 0}^{1}(\zeta, \eta) \subseteq \mathcal{S} \mathscr{P}_{\mu, 0}^{1}(\zeta, \eta)
$$

where $[(0 \leq \zeta<1$ and $1 / 2 \leq \eta)$ or $(\zeta \geq 1$ and $0 \leq \eta<$ 1)].

(2) Consider $\zeta=0$ in Theorem 12; we obtain, for $0 \leq \mu \leq$ $\alpha<1$,

$$
\mathcal{S}_{\alpha, \lambda}^{* n}(0, \eta) \subseteq \mathcal{S}_{\mu, \lambda}^{* n}(0, \eta)
$$

where $0 \leq \eta<1 / 2$.

3.2. Coefficient Bounds. In this subsection, we obtain the coefficient bounds of those functions belonging to the class $\mathcal{S} \mathscr{P}_{\alpha, \lambda}^{n}(\zeta, \eta)$.

Theorem 15. A complex function $f \in \mathscr{A}$ is in $\mathcal{S P P}_{\alpha, \lambda}^{n}(\zeta, \eta)$ if

$$
\begin{aligned}
& \sum_{k=2}^{\infty}[k(1+\zeta)-(\zeta+\eta)][1+\lambda(k-1)]^{2 n} C(\alpha, k)\left|a_{k}\right|^{2} \\
& \quad \leq 1-\eta
\end{aligned}
$$

Proof. It suffices to show that

$$
\begin{aligned}
& \zeta\left|\frac{z\left(\widetilde{D}_{\alpha, \lambda}^{n} f(z)\right)^{\prime}}{\widetilde{D}_{\alpha, \lambda}^{n} f(z)}-1\right|-\Re\left\{\frac{z\left(\widetilde{D}_{\alpha, \lambda}^{n} f(z)\right)^{\prime}}{\widetilde{D}_{\alpha, \lambda}^{n} f(z)}-1\right\} \\
& \quad<1-\eta .
\end{aligned}
$$

We have

$$
\begin{aligned}
& \zeta\left|\frac{z\left(\widetilde{D}_{\alpha, \lambda}^{n} f(z)\right)^{\prime}}{\widetilde{D}_{\alpha, \lambda}^{n} f(z)}-1\right|-\Re\left\{\frac{z\left(\widetilde{D}_{\alpha, \lambda}^{n} f(z)\right)^{\prime}}{\widetilde{D}_{\alpha, \lambda}^{n} f(z)}-1\right\} \\
& \leq(1+\zeta)\left|\frac{z\left(\widetilde{D}_{\alpha, \lambda}^{n} f(z)\right)^{\prime}}{\widetilde{D}_{\alpha, \lambda}^{n} f(z)}-1\right| \\
& \leq \frac{(1+\zeta) \sum_{k=2}^{\infty}(k-1)[1+\lambda(k-1)]^{2 n} C(\alpha, k)\left|a_{k}\right|^{2}|z|^{k-1}}{1-\sum_{k=2}^{\infty}[1+\lambda(k-1)]^{2 n} C(\alpha, k)\left|a_{k}\right|^{2}|z|^{k-1}} \\
& <\frac{(1+\zeta) \sum_{k=2}^{\infty}(k-1)[1+\lambda(k-1)]^{2 n} C(\alpha, k)\left|a_{k}\right|^{2}}{1-\sum_{k=2}^{\infty}[1+\lambda(k-1)]^{2 n} C(\alpha, k)\left|a_{k}\right|^{2}} .
\end{aligned}
$$

Using condition (53), last expression is bounded above by (1$\eta)$.

\section{Uniformly Convex Functions}

This section concerns the class $\mathcal{U} \mathscr{C} \mathscr{V}_{\alpha, \lambda}^{n}(\zeta, \eta)$ and its properties, namely, inclusion relation and coefficient bounds.

4.1. Inclusion Relation. The forthcoming lemma paves the way to provide the inclusion relations in class $\mathcal{U} \mathscr{C} \mathscr{V}_{\alpha, \lambda}^{n}(\zeta, \eta)$.

Lemma 16. Let $R_{\alpha, \lambda}^{n} f(z) \in \mathscr{U} \mathscr{C} \mathscr{V}_{\alpha, \lambda}^{n}(\zeta, \eta)$, and

$$
\sum_{k=2}^{\infty} \frac{k^{2}}{C(\alpha, k)}\left|a_{k}\right|<1 .
$$

Then $f \in \mathscr{U} \mathscr{C} \mathscr{V}_{\alpha, \lambda}^{n}(\zeta, \eta)$. 
Proof. In virtue of Lemma 7, the following implication is done.

$$
\begin{aligned}
R^{\alpha} f(z) \in & \mathcal{U} \mathscr{C} \mathscr{V}_{\alpha, \lambda}^{n}(\zeta, \eta) \\
& \Longleftrightarrow z\left(R^{\alpha} f(z)\right)^{\prime} \in \mathcal{S P}_{\alpha, \lambda}^{n}(\zeta, \eta) \\
& \Longleftrightarrow z\left(R^{\alpha} f\right)^{\prime}(z) \in \mathcal{S} \mathscr{P}_{\alpha, \lambda}^{n}(\zeta, \eta) \\
& \Longrightarrow z f^{\prime}(z) \in \mathcal{S} \mathscr{P}_{\alpha, \lambda}^{n}(\zeta, \eta) \\
& \Longleftrightarrow f(z) \in \mathcal{U} \mathscr{C} \mathscr{V}_{\alpha, \lambda}^{n}(\zeta, \eta) .
\end{aligned}
$$

Therefore, $f(z) \in \mathscr{U} \mathscr{C} \mathscr{V}_{\alpha, \lambda}^{n}(\zeta, \eta)$.

Theorem 17. Let $0 \leq \lambda \leq(1+\zeta) /(1-\eta)$ and

$$
\sum_{k=2}^{\infty} \frac{k^{2}}{C(\alpha, k)}\left|a_{k}\right|<1 .
$$

Then

$$
\mathscr{U} \mathscr{C} \mathscr{V}_{\alpha, \lambda}^{n+1}(\zeta, \eta) \subseteq \mathcal{U} \mathscr{C} \mathscr{V}_{\alpha, \lambda}^{n}(\zeta, \eta)
$$

Proof. In virtue of Lemma 3, the following implication is done.

$$
\begin{aligned}
f(z) \in & \mathcal{U} \mathscr{C} \mathscr{V}_{\alpha, \lambda}^{n+1}(\zeta, \eta) \\
& \Longleftrightarrow z f^{\prime}(z) \in \mathcal{S} \mathscr{P}_{\alpha, \lambda}^{n+1}(\zeta, \eta) \\
& \Longrightarrow z f^{\prime}(z) \in \mathcal{S} \mathscr{P}_{\alpha, \lambda}^{n}(\zeta, \eta) \\
& \Longleftrightarrow f(z) \in \mathcal{U} \mathscr{C} \mathscr{V}_{\alpha, \lambda}^{n}(\zeta, \eta)
\end{aligned}
$$

Therefore, $f(z) \in \mathscr{U} \mathscr{C} \mathscr{V}_{\alpha, \lambda}^{n}(\zeta, \eta)$.

Corollary 18. Let $0 \leq \lambda \leq(1+\zeta) /(1-\eta)$ and

$$
\sum_{k=2}^{\infty} \frac{k^{2}}{C(\alpha, k)}\left|a_{k}\right|<1 .
$$

Then

$$
\mathscr{U} \mathscr{C} \mathscr{V}_{\alpha, \lambda}^{n}(\zeta, \eta) \subseteq \mathscr{U} \mathscr{C} \mathscr{V}_{\alpha, \lambda}(\zeta, \eta)
$$

Proof. The result follows by using Theorem 17.

Remark 19. By giving the parameters $n, \alpha$, and $\zeta$ certain values, new results are obtained as follows.

(1) Consider $\alpha=0$ in Theorem 17; we obtain, for $0 \leq \lambda \leq$ $(1+\zeta) /(1-\eta)$

$$
\mathscr{U} \mathscr{C} \mathscr{V}_{\lambda}^{n+1}(\zeta, \eta) \subseteq \mathcal{U} \mathscr{C} \mathscr{V}_{\lambda}^{n}(\zeta, \eta)
$$

(2) Consider $\zeta=0$ in Theorem 17; we obtain, for $0 \leq \lambda \leq$ $(1+\zeta) /(1-\eta)$

$$
\mathscr{C}_{\alpha, \lambda}^{n+1}(\eta) \subseteq \mathscr{C}_{\alpha, \lambda}^{n}(\eta)
$$

Theorem 20. Let $0 \leq \mu \leq \alpha<1$ and

$$
\sum_{k=2}^{\infty} \frac{k^{2}}{C(\alpha, k)}\left|a_{k}\right|<1 .
$$

Then

$$
\mathcal{U} \mathscr{C} \mathscr{V}_{\alpha, \lambda}^{n}(\zeta, \eta) \subseteq \mathcal{U} \mathscr{C} \mathscr{V}_{\mu, \lambda}^{n}(\zeta, \eta)
$$

where $[(0 \leq \zeta<1$ and $1 / 2 \leq \eta)$ or $(\zeta \geq 1$ and $0 \leq \eta<1)]$.

Proof. The results are obtained using Theorem 12 and apply Alexander relation.

Corollary 21. Let $[(0 \leq \zeta<1$ and $1 / 2 \leq \eta)$ or $(\zeta \geq 1$ and $0 \leq \eta<1)]$. Then

$$
\mathcal{U} \mathscr{C} \mathscr{V}_{\alpha, \lambda}^{n}(\zeta, \eta) \subseteq \mathcal{U} \mathscr{C} \mathscr{V}_{\lambda}(\zeta, \eta)
$$

Corollary 22. Let $0 \leq \mu \leq \alpha<1$. Then

$$
\mathcal{U} \mathscr{C} \mathscr{V}_{\alpha, \lambda}^{n}(\zeta, \eta) \subseteq \mathcal{U} \mathscr{C} \mathscr{V}_{\mu, \lambda}(\zeta, \eta),
$$

where $[(0 \leq \zeta<1$ and $1 / 2 \leq \eta)$ or $(\zeta \geq 1$ and $0 \leq \eta<1)]$.

Remark 23. By giving the parameters $n, \alpha, \lambda$, and $\zeta$ certain values, we obtain new results as follows.

(1) Consider $n=1$ and $\lambda=0$ in Theorem 20; we obtain for $0 \leq \mu \leq \alpha<1$,

$$
\mathcal{U} \mathscr{C} \mathscr{V}_{\alpha, 0}^{1}(\zeta, \eta) \subseteq \mathcal{U} \mathscr{C} \mathscr{V}_{\mu, 0}^{1}(\zeta, \eta)
$$

where $[(0 \leq \zeta<1$ and $1 / 2 \leq \eta)$ or $(\zeta \geq 1$ and $0 \leq \eta<$ 1)].

(2) Consider $\zeta=0$ in Theorem 20; we obtain for $0 \leq \mu \leq$ $\alpha<1$,

$$
\mathscr{C}_{\alpha, \lambda}^{n}(0, \eta) \subseteq \mathscr{C}_{\mu, \lambda}^{n}(0, \eta)
$$

where $0 \leq \eta<1 / 2$.

4.2. Coefficient Bounds. In this subsection, we obtain the coefficient bounds of those functions belonging to the class $\mathscr{U} \mathscr{C} \mathscr{V}_{\alpha, \lambda}^{n}(\zeta, \eta)$

Theorem 24. A complex function $f \in \mathscr{A}$ is in $\mathscr{U} \mathscr{C} \mathscr{V}_{\alpha, \lambda}^{n}(\zeta, \eta)$ if

$$
\begin{aligned}
& \sum_{k=2}^{\infty} k[k(1+\zeta)-(\zeta+\eta)][1+\lambda(k-1)]^{2 n} C(\alpha, k)\left|a_{k}\right|^{2} \\
& \quad \leq 1-\eta
\end{aligned}
$$

Proof. The result follows from Theorem 15 and the following relation:

$$
\begin{aligned}
& f \in \mathcal{U} \mathscr{C} \mathscr{V}_{\alpha, \lambda}^{n}(\zeta, \eta) \Longleftrightarrow \\
& z f^{\prime} \in \mathcal{S}_{\alpha, \lambda}^{n}(\zeta, \eta) .
\end{aligned}
$$




\section{Conclusion}

This paper introduced two classes of uniformly geometric functions of order $\eta$ type $\zeta$. Literally speaking, convex and starlike uniformly functions of order $\eta$ type $\zeta$ were introduced by involving the constructed differential operator $\widetilde{D}_{\alpha, \lambda}^{n}$. Also, the geometric interpretation of these functions was given. Finally, two properties of each class were investigated, namely, inclusion relations and coefficient bounds.

\section{Conflicts of Interest}

The authors declare that they have no conflicts of interest regarding the publication of this paper.

\section{Acknowledgments}

The work here is supported by MOHE Grant FRGS/1/2016/ STG06/UKM/01/1.

\section{References}

[1] M. Darus and K. Al-Shaqsi, "Differential sandwich theorems with generalised derivative operator," International Journal of Computational and Mathematical Sciences, vol. 2, no. 2, pp. 7578, 2008.

[2] F. M. Al-Oboudi, "On univalent functions defined by a generalized Sălăgean operator," International Journal of Mathematics and Mathematical Sciences, vol. 2004, no. 27, pp. 1429-1436, 2004.

[3] M. I. Robertson, "On the theory of univalent functions," Annals of Mathematics. Second Series, vol. 37, no. 2, pp. 374-408, 1936.

[4] R. Bharati, R. Parvatham, and A. Swaminathan, "On subclasses of uniformly convex functions and corresponding class of starlike functions," Tamkang Journal of Mathematics, vol. 28, no. 1, pp. 17-32, 1997.

[5] A. W. Goodman, "On uniformly starlike functions," Journal of Mathematical Analysis and Applications, vol. 155, no. 2, pp. 364370, 1991.

[6] F. Rønning, "Uniformly convex functions and a corresponding class of starlike functions," Proceedings of the American Mathematical Society, vol. 118, no. 1, pp. 189-196, 1993.

[7] F. Rønning, "On starlike functions associated with parabolic regions," Annales Universitatis Mariae Curie-Sklodowska. Sectio A. Mathematica, vol. 45, pp. 117-122, 1991.

[8] S. Kanas and A. Wisniowska, "Conic regions and k-uniform convexity," Journal of Computational and Applied Mathematics, vol. 105, no. 1-2, pp. 327-336, 1999.

[9] S. A. Kanas and A. Wisniowska, "Conic domains and starlike functions," Revue Roumaine de Mathématiques Pures et Appliquées, vol. 45, no. 4, pp. 647-657, 2000.

[10] F. M. Al-Oboudi and K. A. Al-Amoudi, "On classes of analytic functions related to conic domains," Journal of Mathematical Analysis and Applications, vol. 339, no. 1, pp. 655-667, 2008.

[11] S. Ruscheweyh and T. Sheil-Small, "Hadamard products of Schlicht functions and the Pólya-Schoenberg conjecture," Commentarii Mathematici Helvetici, vol. 48, no. 1, pp. 119-135, 1973.

[12] S. Ruscheweyh, Convolutions in Geometric Function Theory, Gaetan Morin Editeur Ltee, 1982.
[13] S. S. Miller and P. T. Mocanu, Differential Subordinations: Theory and Applications, vol. 225 of Monographs and Textbooks in Pure and Applied Mathematics, CRC Press, Boca Raton, Fla, USA, 2000. 


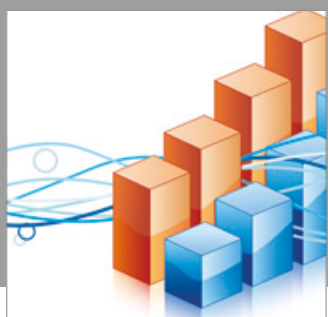

Advances in

Operations Research

vatersals

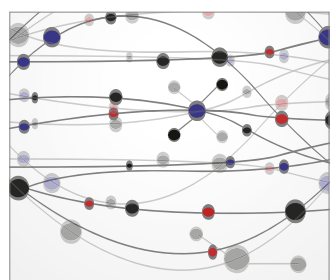

\section{The Scientific} World Journal
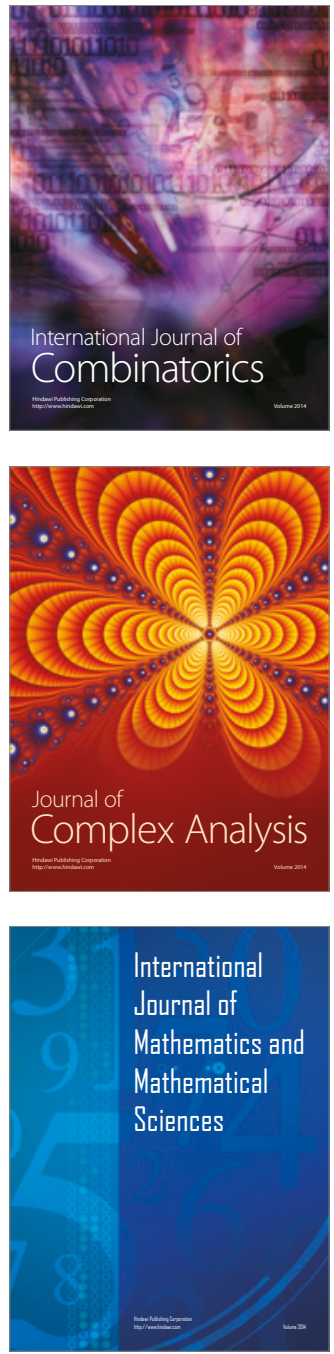
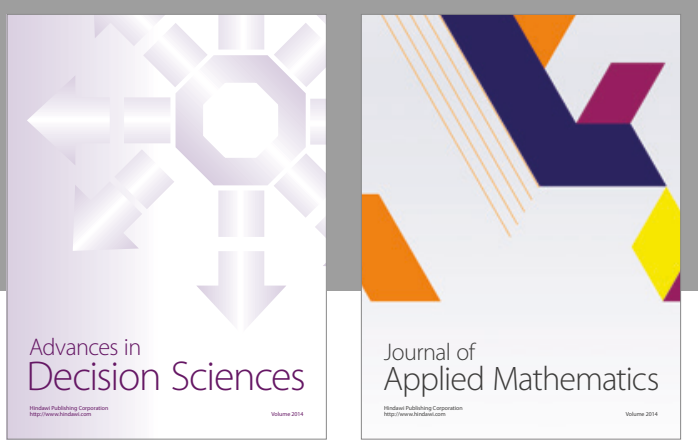

Algebra

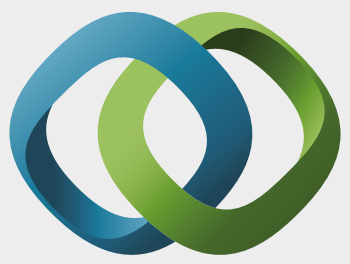

\section{Hindawi}

Submit your manuscripts at

https://www.hindawi.com
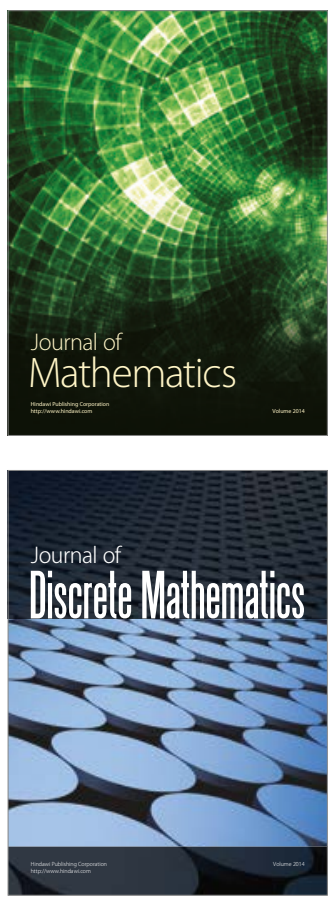

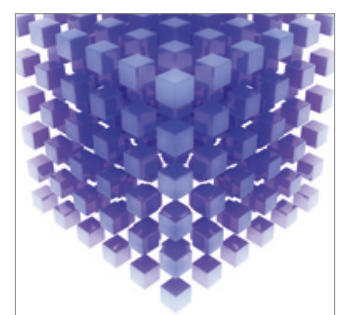

Mathematical Problems in Engineering
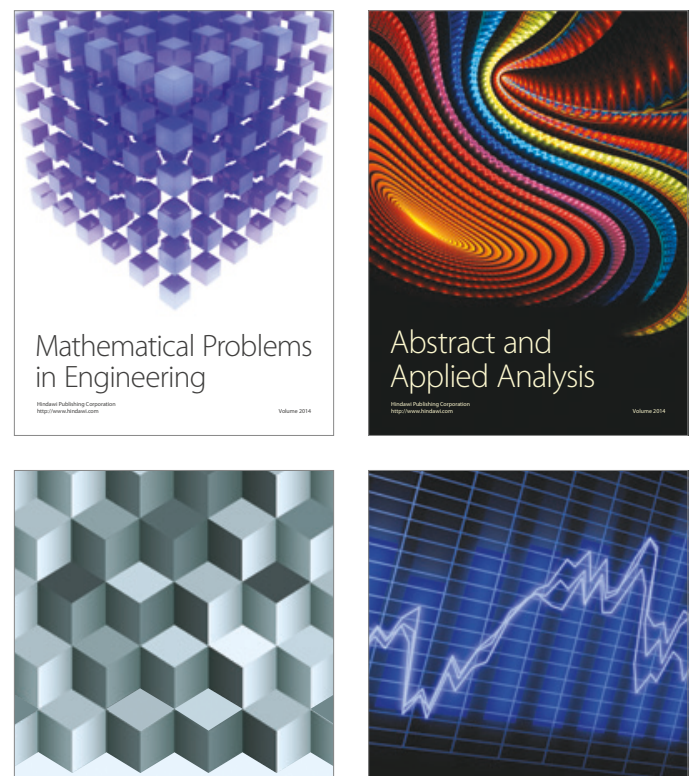

Journal of

Function Spaces

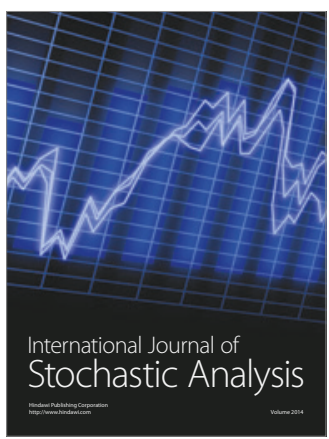

Probability and Statistics
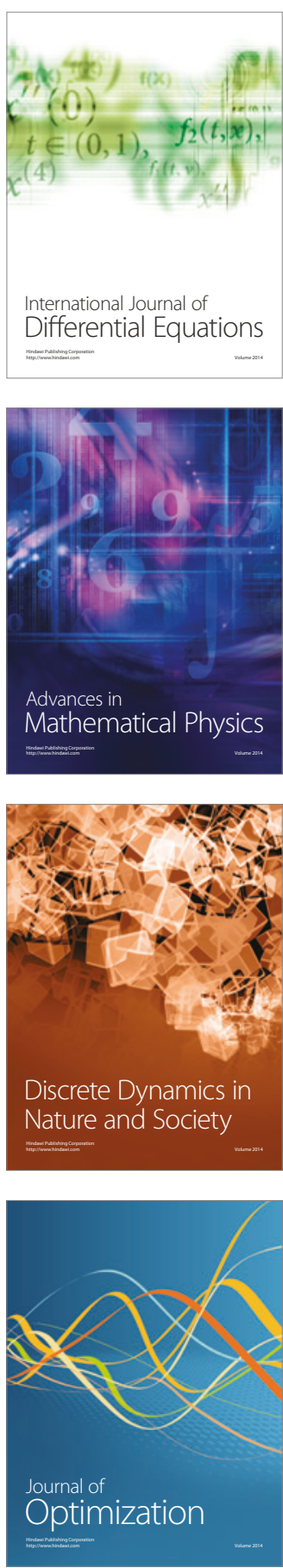